\title{
Stability Analysis of One-Leg Methods for Nonlinear Neutral Delay Integrodifferential Equations
}

\author{
Yuexin Yu and Liping Wen \\ Department of Mathematics, Xiangtan University, Xiangtan 411105, China \\ Correspondence should be addressed to Yuexin Yu; yuyx@xtu.edu.cn
}

Received 8 April 2015; Revised 16 June 2015; Accepted 24 June 2015

Academic Editor: Antonia Vecchio

Copyright ( 2015 Y. Yu and L. Wen. This is an open access article distributed under the Creative Commons Attribution License, which permits unrestricted use, distribution, and reproduction in any medium, provided the original work is properly cited.

This paper is concerned with the numerical solution of nonlinear neutral delay integrodifferential equations (NDIDEs). The adaptation of one-leg methods is considered. It is proved that an $A$-stable one-leg method can preserve the global stability and a strongly $A$-stable one-leg method can preserve the asymptotic stability of the analytical solution of nonlinear NDIDEs. Numerical tests are given to confirm the theoretical results.

\section{Introduction}

In this paper, we consider the initial value problem (IVP) of nonlinear neutral delay integrodifferential equations:

$$
\begin{gathered}
y^{\prime}(t)=f\left(t, y(t), y(t-\tau), y^{\prime}(t-\tau),\right. \\
\left.\int_{t-\tau}^{t} g(t, \xi, y(\xi)) d \xi\right), \quad t \geq 0, \\
y(t)=\varphi(t), \quad-\tau \leq t \leq 0,
\end{gathered}
$$

where $\tau>0$ is a constant delay, $\varphi:[-\tau, 0] \rightarrow \mathbb{C}^{N}$ is a given continuously differential function, and $f:[0,+\infty) \times \mathbb{C}^{N} \times$ $\mathbb{C}^{N} \times \mathbb{C}^{N} \times \mathbb{C}^{N} \rightarrow \mathbb{C}^{N}$ and $g:[0,+\infty) \times \mathbb{R} \times \mathbb{C}^{N} \rightarrow \mathbb{C}^{N}$ are given continuous mappings.

Neutral delay integrodifferential equations (NDIDEs) arise widely in scientific and engineering fields such as physics, biology, medicine, economics, and control system (see [1-3] and the references therein). Generally speaking, it is difficult to obtain the analytical solutions of such equations. In view of this, people began to study the numerical solutions of the equations. For the special cases of NDIDEs, such as delay differential equations, delay integrodifferential equations, and neutral delay differential equations, the theory of computational methods has been studied by many authors and a great deal of interesting results have been found in recent 30 years. But, for NDIDEs, only a few results have been presented in the literature. In 2005, Zhao et al. [4] discussed the asymptotic stability of analytical solution and numerical solution (obtained by linear $\theta$-methods and BDF methods) of linear neutral Volterra delay integrodifferential system:

$$
\begin{aligned}
& A u^{\prime}(t)+B u(t)+C u^{\prime}(t-\tau)+D u(t-\tau) \\
& +G \int_{t-\tau}^{t} u(x) d x=0, \quad t>0, \\
& u(t)=\phi(t), \quad-\tau \leq t \leq 0,
\end{aligned}
$$

where $A, B, C, D, G \in \mathbb{R}^{d \times d}, \tau>0$ and the matrix $A$ may be singular. Later, $\mathrm{Xu}$ and Zhao [5] further considered the asymptotic stability of Runge-Kutta methods for system (2). In 2008, Zhang and Vandewalle [6] dealt with the asymptotic stability of exact and discrete solutions of neutral multidelay integrodifferential equations. Sufficient conditions for the asymptotic stability of the analytical solution have been derived, and the asymptotic stability criteria of Runge-Kutta methods and linear multistep methods were constructed. $\mathrm{Wu}$ and Gan [7] investigated a test equation for onedimension linear NDIDEs and got some delay-dependent stability results.

For the nonlinear NDIDEs (1), Yu and Li [8] discussed the global stability and asymptotic stability of $(k, l)$ algebraically stable Runge-Kutta methods. Recently, Hu and Huang [9] 
considered the analytical and numerical stability of nonlinear NDIDEs. Sufficient conditions for the analytical stability of nonlinear NDIDEs are derived, and they proved that any $A$ stable linear multistep method can preserve the asymptotic stability of the analytical solution of nonlinear NDIDEs (1). For another case of nonlinear NDIDEs, namely, the NDIDEs of "Hale's form"

$$
\begin{aligned}
& {[y(t)-N y(t-\tau)]^{\prime}} \\
& =f\left(t, y(t), y(t-\tau), \int_{t-\tau}^{t} g(t, \xi, y(\xi)) d \xi\right), \\
& y(t)=\varphi(t), \quad-\tau \leq t \leq 0,
\end{aligned}
$$

Yu et al. [10] and Zhang et al. [11, 12] investigated the stability of Runge-Kutta methods and one-leg methods, respectively.

In this paper we are interested in the stability of oneleg methods for nonlinear NDIDEs (1). It is proved that an $A$-stable one-leg method can preserve the global stability and a strongly $A$-stable one-leg method can preserve the asymptotic stability of the analytical solution of nonlinear NDIDEs. Numerical tests are given to confirm the theoretical results in the end.

\section{Problem Class $\mathrm{D}\left(\alpha, \beta_{1}, \beta_{2}, \beta_{3}, \gamma, \delta\right)$ and Its Stability}

Let $\langle\cdot, \cdot\rangle$ denote the inner product and $\|\cdot\|$ the corresponding norm in space $\mathbb{C}^{N}$. Assume that the continuous mappings $f$ and $g$ in problem (1) satisfy the following conditions:

$$
\begin{aligned}
& \operatorname{Re}\left\langle u_{1}-u_{2}, f\left(t, u_{1}, v, w, x\right)-f\left(t, u_{2}, v, w, x\right)\right\rangle \\
& \leq \alpha\left\|u_{1}-u_{2}\right\|^{2}, \quad \forall t \geq 0, u_{1}, u_{2}, v, w, x \in \mathbb{C}^{N}, \\
& \left\|f\left(t, u, v_{1}, w_{1}, x_{1}\right)-f\left(t, u, v_{2}, w_{2}, x_{2}\right)\right\| \\
& \leq \beta_{1}\left\|v_{1}-v_{2}\right\|+\beta_{2}\left\|w_{1}-w_{2}\right\|+\beta_{3}\left\|x_{1}-x_{2}\right\|, \\
& \forall t \geq 0, u, v_{1}, v_{2}, w_{1}, w_{2}, x_{1}, x_{2} \in \mathbb{C}^{N}, \\
& \left\|g\left(t, \theta, s_{1}\right)-g\left(t, \theta, s_{2}\right)\right\| \leq \gamma\left\|s_{1}-s_{2}\right\|, \\
& \forall t \geq 0, t-\tau \leq \theta \leq t, s_{1}, s_{2} \in \mathbb{C}^{N},
\end{aligned}
$$

where $\alpha, \beta_{1}, \beta_{2}, \beta_{3}$, and $\gamma$ are real constants and $\beta_{2}<1$. Furthermore, we also consider the function $F(t, u, v, w, x, r, s):=$ $f(t, u, v, f(t-\tau, v, w, x, r), s)$ and assume that it is continuous and satisfies

$$
\begin{aligned}
& \left\|F\left(t, u, v_{1}, w, x, r, s\right)-F\left(t, u, v_{2}, w, x, r, s\right)\right\| \\
& \quad \leq \delta\left\|v_{1}-v_{2}\right\|, \quad \forall t \geq \tau, u, v_{1}, v_{2}, w, x, r, s \in \mathbb{C}^{N},
\end{aligned}
$$

where $\delta>0$ is a real constant.

Throughout this paper, we assume that problem (1) has a unique exact solution $y(t)$, and we use the symbol $\mathrm{D}\left(\alpha, \beta_{1}, \beta_{2}, \beta_{3}, \gamma, \delta\right)$ to denote the problem class consisting of all of problem (1) satisfying conditions (4)-(7).
Remark 1. When the right-hand side function of problem (1) does not possess the term $y^{\prime}(t-\tau)$, problem (1) degenerates into an IVP of delay integrodifferential equations (DIDEs):

$$
\begin{aligned}
& y^{\prime}(t)=f\left(t, y(t), y(t-\tau), \int_{t-\tau}^{t} g(t, \xi, y(\xi)) d \xi\right), \\
& t \geq 0, \\
& y(t)=\varphi(t), \quad-\tau \leq t \leq 0 .
\end{aligned}
$$

The stability of numerical methods for DIDEs has been investigated in [13-17].

Remark 2. When the right-hand side function of problem (1) does not possess the integral term, problem (1) degenerates into an IVP of neutral delay differential equations (NDDEs):

$$
\begin{aligned}
y^{\prime}(t) & =f\left(t, y(t), y(t-\tau), y^{\prime}(t-\tau)\right), \quad t \geq 0, \\
y(t) & =\varphi(t), \quad-\tau \leq t \leq 0 .
\end{aligned}
$$

The stability of numerical methods for NDDEs has been researched in [18-22].

Remark 3. When the right-hand side function of problem (1) does not possess the term $y^{\prime}(t-\tau)$ and the integral term, problem (1) degenerates into an IVP of delay differential equations (DDEs):

$$
\begin{aligned}
y^{\prime}(t) & =f(t, y(t), y(t-\tau)), \quad t \geq 0, \\
y(t) & =\varphi(t), \quad-\tau \leq t \leq 0
\end{aligned}
$$

The stability results of numerical methods for DDEs can be found in [23-28] and so forth.

For problems of the class $\mathrm{D}\left(\alpha, \beta_{1}, \beta_{2}, \beta_{3}, \gamma, \delta\right), \mathrm{Hu}$ and Huang derived the following stability results (see [9]).

Theorem 4. Suppose problem (1) belongs to the class $\mathrm{D}\left(\alpha, \beta_{1}, \beta_{2}, \beta_{3}, \gamma, \delta\right)$ satisfying $\alpha+\left(\delta+\beta_{3} \gamma \tau\right) /\left(1-\beta_{2}\right) \leq 0$. Then one has

$$
\|y(t)-z(t)\| \leq \max \{\|\varphi(0)-\psi(0)\|, \kappa\}, \quad \forall t>0,
$$

where

$\kappa$

$$
=\sup _{-\tau \leq t \leq 0} \frac{\left(\beta_{1}+\beta_{3} \gamma \tau\right)\|\varphi(t)-\psi(t)\|+\beta_{2}\left\|\varphi^{\prime}(t)-\psi^{\prime}(t)\right\|}{-\alpha}
$$

and $z(t)$ denotes the solution of any given perturbed problem of (1):

$$
\begin{gathered}
z^{\prime}(t)=f\left(t, z(t), z(t-\tau), z^{\prime}(t-\tau)\right. \\
\left.\int_{t-\tau}^{t} g(t, \xi, z(\xi)) d \xi\right), \quad t \geq 0 \\
z(t)=\psi(t), \quad-\tau \leq t \leq 0
\end{gathered}
$$

where $\psi:[-\tau, 0] \rightarrow \mathbb{C}^{N}$ is a given continuously differential function. 
Theorem 5. Suppose problem (1) belongs to the class $\mathrm{D}\left(\alpha, \beta_{1}, \beta_{2}, \beta_{3}, \gamma, \delta\right)$ satisfying $\alpha+\left(\delta+\beta_{3} \gamma \tau\right) /\left(1-\beta_{2}\right)<0$. Then one has

$$
\lim _{t \rightarrow+\infty}\|y(t)-z(t)\|=0
$$

Inequality (11) characterizes the stability property and relation (14) characterizes the asymptotic stability property of problem (1), respectively.

\section{Stability Analysis of One-Leg Methods for NDIDEs}

Consider using a one-leg $k$-step method (for ordinary differential equations)

$$
\rho(E) y_{n}=h f\left(\sigma(E) t_{n}, \sigma(E) y_{n}\right), \quad n=0,1,2, \ldots,
$$

to solve problem (1); we have

$$
\begin{array}{r}
\rho(E) y_{n}=h f\left(\sigma(E) t_{n}, \sigma(E) y_{n}, \sigma(E) y_{n-m}, \tilde{y}_{n}, G_{n}\right), \\
n=0,1,2, \ldots,
\end{array}
$$

where $h=\tau / m, m$ is an arbitrarily given positive integer, $t_{n}=n h, E$ is the translation operator, $E y_{n}=y_{n+1}, y_{n}$ is an approximation to $y\left(t_{n}\right), y_{n}=\varphi\left(t_{n}\right)$ for $n \leq 0$, and $G_{n}$ is an approximation to $\int_{\sigma(E) t_{n}-\tau}^{\sigma(E) t_{n}} g\left(\sigma(E) t_{n}, \xi, y(\xi)\right) d \xi$, which can be computed by the repeated trapezoidal rule:

$$
\begin{aligned}
G_{n} & =h\left[\sum_{k=1}^{m-1} g\left(\sigma(E) t_{n}, \sigma(E) t_{n-k}, \sigma(E) y_{n-k}\right)\right. \\
& +\frac{1}{2} g\left(\sigma(E) t_{n}, \sigma(E) t_{n}, \sigma(E) y_{n}\right) \\
& \left.+\frac{1}{2} g\left(\sigma(E) t_{n}, \sigma(E) t_{n-m}, \sigma(E) y_{n-m}\right)\right] .
\end{aligned}
$$

$\tilde{y}_{n}$ is an approximation to $y^{\prime}\left(\sigma(E) t_{n}-\tau\right)$, which is obtained by using the following formula:

$$
\begin{aligned}
\tilde{y}_{n} & =f\left(\sigma(E) t_{n}\right. \\
& \left.-\tau, \sigma(E) y_{n-m}, \sigma(E) y_{n-2 m}, \tilde{y}_{n-m}, G_{n-m}\right),
\end{aligned}
$$

where $\tilde{y}_{n}=\varphi^{\prime}\left(\sigma(E) t_{n}-\tau\right)$ for $\sigma(E) t_{n}-\tau<0$ and $\rho(x)=$ $\sum_{j=0}^{k} \alpha_{j} x^{j}$ and $\sigma(x)=\sum_{j=0}^{k} \beta_{j} x^{j}$ are generating polynomials which are assumed to have real coefficients and no common divisor. We also assume $\rho(1)=0, \rho^{\prime}(1)=\sigma(1)=1$, and $\sigma^{\prime}(1) \geq 0$.
Similarly, applying the same method to perturbed problem (13), we have

$$
\begin{aligned}
& \rho(E) z_{n}=h f\left(\sigma(E) t_{n}, \sigma(E) z_{n}, \sigma(E) z_{n-m}, \widetilde{z}_{n}, H_{n}\right), \\
& n=0,1,2, \ldots, \\
& H_{n}=h\left[\sum_{k=1}^{m-1} g\left(\sigma(E) t_{n}, \sigma(E) t_{n-k}, \sigma(E) z_{n-k}\right)\right. \\
& \quad+\frac{1}{2} g\left(\sigma(E) t_{n}, \sigma(E) t_{n}, \sigma(E) z_{n}\right) \\
& \left.\quad+\frac{1}{2} g\left(\sigma(E) t_{n}, \sigma(E) t_{n-m}, \sigma(E) z_{n-m}\right)\right],
\end{aligned}
$$

where $z_{n}$ and $H_{n}$ are approximations to $z\left(t_{n}\right)$ and $\int_{\sigma(E) t_{n}-\tau}^{\sigma(E) t_{n}} g\left(\sigma(E) t_{n}, \xi, z(\xi)\right) d \xi$, respectively, $z_{n}=\psi\left(t_{n}\right)$ for $n \leq 0$, and $\widetilde{z}_{n}$ is an approximation to $z^{\prime}\left(\sigma(E) t_{n}-\tau\right)$, which can be computed by

$$
\begin{aligned}
\widetilde{z}_{n} & =f\left(\sigma(E) t_{n}\right. \\
& \left.-\tau, \sigma(E) z_{n-m}, \sigma(E) z_{n-2 m}, \widetilde{z}_{n-m}, H_{n-m}\right),
\end{aligned}
$$

where $\widetilde{z}_{n}=\psi^{\prime}\left(\sigma(E) t_{n}-\tau\right)$ for $\sigma(E) t_{n}-\tau<0$.

For a real symmetric positive $k \times k$ matrix $G=\left[g_{i j}\right]$, the norm $\|\cdot\|_{G}$ is defined by

$$
\begin{aligned}
\|U\|_{G}=\left(\sum_{i, j=1}^{k} g_{i j}\left\langle u_{i}, u_{j}\right\rangle\right)^{1 / 2}, & \\
\forall U & =\left[u_{1}^{T}, u_{2}^{T}, \ldots, u_{k}^{T}\right]^{T} \in \mathbb{C}^{k N} .
\end{aligned}
$$

Theorem 6. Assume that one-leg method (15) is A-stable. Then the numerical solutions $\left\{y_{n}\right\}$ and $\left\{z_{n}\right\}$, obtained by using corresponding method (16) to problems (1) and (13) which belong to the class $\mathrm{D}\left(\alpha, \beta_{1}, \beta_{2}, \beta_{3}, \gamma, \delta\right)$ with $\alpha+\left(\delta+\beta_{3} \gamma \tau\right) /(1-$ $\left.\beta_{2}\right) \leq 0$, respectively, satisfy the global stability inequality

$$
\begin{aligned}
&\left\|y_{n}-z_{n}\right\| \leq C \max \left\{\max _{0 \leq j \leq k-1}\left\|y_{j}-z_{j}\right\|, \Gamma\right\} \\
& n=1,2,3, \ldots
\end{aligned}
$$

for all $h>0$, where $C$ depends only on the method, $\beta_{1}, \beta_{2}, \beta_{3}$, $\gamma, \delta$, and $\tau$, and

$$
\Gamma=\max _{-\tau \leq t \leq 0}\left\{\|\varphi(t)-\psi(t)\|,\left\|\varphi^{\prime}(t)-\psi^{\prime}(t)\right\|\right\} .
$$

Proof. Let

$$
\begin{aligned}
w_{n} & =y_{n}-z_{n}, \\
W_{n} & =\left(w_{n}^{T}, w_{n+1}^{T}, \ldots, w_{n+k-1}^{T}\right)^{T}, \\
q_{n} & =\left\lfloor\frac{n+\sigma^{\prime}(1)}{m}\right\rfloor,
\end{aligned}
$$


where $\lfloor\cdot\rfloor$ denotes the integer part; then $q_{n} \tau \leq \sigma(E) t_{n}<\left(q_{n}+\right.$ 1) $\tau$.

Since $A$-stability is equivalent to $G$-stability (cf. [29]), there is a $k \times k$ real symmetric positive definite matrix $G$ such that, for any real sequence $\left\{a_{i}\right\}_{i=0}^{k}$, the following inequality holds:

$$
A_{1}^{T} G A_{1}-A_{0}^{T} G A_{0} \leq 2 \sigma(E) a_{0} \rho(E) a_{0},
$$

where $A_{i}=\left(a_{i}, a_{i+1}, \ldots, a_{i+k-1}\right)^{T}(i=0,1)$. Therefore, we can easily obtain (cf. [29])

$$
\left\|W_{n+1}\right\|_{G}^{2} \leq\left\|W_{n}\right\|_{G}^{2}+2 \operatorname{Re}\left\langle\sigma(E) w_{n}, \rho(E) w_{n}\right\rangle .
$$

Using condition (4), we have

$$
\begin{aligned}
2 \operatorname{Re} & \left\langle\sigma(E) w_{n}, \rho(E) w_{n}\right\rangle=2 h \operatorname{Re}\langle\sigma(E) \\
\cdot & w_{n}, f\left(\sigma(E) t_{n}, \sigma(E) y_{n}, \sigma(E) y_{n-m}, \tilde{y}_{n}, G_{n}\right) \\
- & \left.f\left(\sigma(E) t_{n}, \sigma(E) z_{n}, \sigma(E) z_{n-m}, \widetilde{z}_{n}, H_{n}\right)\right\rangle \\
& \leq 2 h \alpha\left\|\sigma(E) w_{n}\right\|^{2}+2 h\left\|\sigma(E) w_{n}\right\| \\
& \cdot \| f\left(\sigma(E) t_{n}, \sigma(E) z_{n}, \sigma(E) y_{n-m}, \tilde{y}_{n}, G_{n}\right) \\
& -f\left(\sigma(E) t_{n}, \sigma(E) z_{n}, \sigma(E) z_{n-m}, \widetilde{z}_{n}, H_{n}\right) \| .
\end{aligned}
$$

When $\sigma(E) t_{n}-\tau<0$, that is, $q_{n}=0$, (27) leads to

$$
\begin{aligned}
& 2 \operatorname{Re}\left\langle\sigma(E) w_{n}, \rho(E) w_{n}\right\rangle \\
& \leq 2 h \alpha\left\|\sigma(E) w_{n}\right\|^{2} \\
& \quad+2 h\left\|\sigma(E) w_{n}\right\|\left[\left(\beta_{1}+\beta_{2}\right) \Gamma+\beta_{3}\left\|G_{n}-H_{n}\right\|\right] .
\end{aligned}
$$

When $\sigma(E) t_{n}-\tau \geq 0$, that is, $q_{n} \geq 1$, using conditions (4)-(7), (27) leads to

$$
\begin{aligned}
& 2 \operatorname{Re}\left\langle\sigma(E) w_{n}, \rho(E) w_{n}\right\rangle \leq 2 h \alpha\left\|\sigma(E) w_{n}\right\|^{2}+2 h \| \sigma(E) \\
& \cdot w_{n} \|\left[\| f\left(\sigma(E) t_{n}, \sigma(E) z_{n}, \sigma(E) y_{n-m},\right.\right. \\
& f\left(\sigma(E) t_{n}-\tau, \sigma(E) y_{n-m}, \sigma(E) y_{n-2 m}, \tilde{y}_{n-m}, G_{n-m}\right), \\
& \left.G_{n}\right)-f\left(\sigma(E) t_{n}, \sigma(E) z_{n}, \sigma(E) z_{n-m},\right. \\
& f\left(\sigma(E) t_{n}-\tau, \sigma(E) z_{n-m}, \sigma(E) z_{n-2 m}, \tilde{z}_{n-m}, H_{n-m}\right), \\
& \left.\left.H_{n}\right) \|\right] \leq 2 h \alpha\left\|\sigma(E) w_{n}\right\|^{2}+2 h\left\|\sigma(E) w_{n}\right\|[\delta \| \sigma(E) \\
& \cdot w_{n-m}\left\|+\beta_{3}\right\| G_{n}-H_{n}\left\|+\beta_{2}\right\| f\left(\sigma(E) t_{n}-\tau,\right. \\
& \left.\sigma(E) z_{n-m}, \sigma(E) y_{n-2 m}, \tilde{y}_{n-m}, G_{n-m}\right)-f\left(\sigma(E) t_{n}\right. \\
& \left.\left.-\tau, \sigma(E) z_{n-m}, \sigma(E) z_{n-2 m}, \widetilde{z}_{n-m}, H_{n-m}\right) \|\right]
\end{aligned}
$$

$$
\begin{aligned}
& \leq 2 h \alpha\left\|\sigma(E) w_{n}\right\|^{2}+2 h\left\|\sigma(E) w_{n}\right\|\left[\delta \sum_{j=1}^{q_{n}} \beta_{2}^{j-1} \| \sigma(E)\right. \\
& \cdot w_{n-j m}\left\|+\beta_{3} \sum_{j=0}^{q_{n}-1} \beta_{2}^{j}\right\| G_{n-j m}-H_{n-j m} \|+\beta_{2}^{q_{n}}\left(\left(\beta_{1}\right.\right. \\
& \left.\left.\left.+\beta_{2}\right) \Gamma+\beta_{3}\left\|G_{n-q_{n} m}-H_{n-q_{n} m}\right\|\right)\right]=2 h \alpha \| \sigma(E) \\
& \cdot w_{n}\left\|^{2}+2 h\right\| \sigma(E) w_{n} \|\left[\delta \sum_{j=1}^{q_{n}} \beta_{2}^{j-1}\left\|\sigma(E) w_{n-j m}\right\|\right. \\
& \left.+\beta_{3} \sum_{j=0}^{q_{n}} \beta_{2}^{j}\left\|G_{n-j m}-H_{n-j m}\right\|+\beta_{2}^{q_{n}}\left(\beta_{1}+\beta_{2}\right) \Gamma\right],
\end{aligned}
$$

where, here and below, we define $\sum_{k=s}^{t}$ equal to 0 for $t<s$. Combining (28) and (29) yields

$$
\begin{aligned}
& 2 \operatorname{Re}\left\langle\sigma(E) w_{n}, \rho(E) w_{n}\right\rangle \leq 2 h \alpha\left\|\sigma(E) w_{n}\right\|^{2} \\
& +2 h\left\|\sigma(E) w_{n}\right\|\left[\delta \sum_{j=1}^{q_{n}} \beta_{2}^{j-1}\left\|\sigma(E) w_{n-j m}\right\|\right. \\
& \left.+\beta_{3} \sum_{j=0}^{q_{n}} \beta_{2}^{j}\left\|G_{n-j m}-H_{n-j m}\right\|+\beta_{2}^{q_{n}}\left(\beta_{1}+\beta_{2}\right) \Gamma\right] .
\end{aligned}
$$

Substituting (30) into (26) and using condition $\alpha+(\delta+$ $\left.\beta_{3} \gamma \tau\right) /\left(1-\beta_{2}\right) \leq 0$, we obtain

$$
\begin{aligned}
& \left\|W_{n+1}\right\|_{G}^{2} \leq\left\|W_{n}\right\|_{G}^{2}+2 h \alpha\left\|\sigma(E) w_{n}\right\|^{2}+2 h\left\|\sigma(E) w_{n}\right\| \\
& .\left[\delta \sum_{j=1}^{q_{n}} \beta_{2}^{j-1}\left\|\sigma(E) w_{n-j m}\right\|\right. \\
& \left.+\beta_{3} \sum_{j=0}^{q_{n}} \beta_{2}^{j}\left\|G_{n-j m}-H_{n-j m}\right\|+\beta_{2}^{q_{n}}\left(\beta_{1}+\beta_{2}\right) \Gamma\right] \\
& \leq\left\|W_{n}\right\|_{G}^{2}+2 h \alpha\left\|\sigma(E) w_{n}\right\|^{2}+h \delta \sum_{j=0}^{q_{n}} \beta_{2}^{j}\left\|\sigma(E) w_{n}\right\|^{2} \\
& +h \delta \sum_{j=1}^{q_{n}} \beta_{2}^{j-1}\left\|\sigma(E) w_{n-j m}\right\|^{2}+h \beta_{2}^{q_{n}} \frac{\left(\beta_{1}+\beta_{2}\right)^{2}}{\delta} \Gamma^{2} \\
& +2 h^{2} \beta_{3} \gamma\left\|\sigma(E) w_{n}\right\| \sum_{j=0}^{q_{n}} \beta_{2}^{j}\left(\sum_{k=1}^{m-1}\left\|\sigma(E) w_{n-j m-k}\right\|\right. \\
& \left.+\frac{1}{2}\left\|\sigma(E) w_{n-j m}\right\|+\frac{1}{2}\left\|\sigma(E) w_{n-(j+1) m}\right\|\right)
\end{aligned}
$$




$$
\begin{aligned}
& \leq\left\|W_{n}\right\|_{G}^{2}+h\left(2 \alpha+\frac{\delta}{1-\beta_{2}}+\frac{\beta_{3} \gamma \tau}{1-\beta_{2}}\right)\left\|\sigma(E) w_{n}\right\|^{2} \\
& +h \delta \sum_{j=1}^{q_{n}} \beta_{2}^{j-1}\left\|\sigma(E) w_{n-j m}\right\|^{2}+h \beta_{2}^{q_{n}} \frac{\left(\beta_{1}+\beta_{2}\right)^{2}}{\delta} \Gamma^{2} \\
& +h^{2} \beta_{3} \gamma \sum_{j=0}^{q_{n}} \beta_{2}^{j}\left(\sum_{k=1}^{m-1}\left\|\sigma(E) w_{n-j m-k}\right\|^{2}\right. \\
& \left.+\frac{1}{2}\left\|\sigma(E) w_{n-j m}\right\|^{2}+\frac{1}{2}\left\|\sigma(E) w_{n-(j+1) m}\right\|^{2}\right) \\
& \leq\left\|W_{n}\right\|_{G}^{2}+h \alpha\left\|\sigma(E) w_{n}\right\|^{2} \\
& +h \delta \sum_{j=1}^{q_{n}} \beta_{2}^{j-1}\left\|\sigma(E) w_{n-j m}\right\|^{2}+h \beta_{2}^{q_{n}} \frac{\left(\beta_{1}+\beta_{2}\right)^{2}}{\delta} \Gamma^{2} \\
& +h^{2} \beta_{3} \gamma \sum_{j=0}^{q_{n}} \beta_{2}^{j}\left(\sum_{k=1}^{m-1}\left\|\sigma(E) w_{n-j m-k}\right\|^{2}\right. \\
& \left.+\frac{1}{2}\left\|\sigma(E) w_{n-j m}\right\|^{2}+\frac{1}{2}\left\|\sigma(E) w_{n-(j+1) m}\right\|^{2}\right)
\end{aligned}
$$

By induction, (31) gives

$$
\begin{aligned}
\left\|W_{n+1}\right\|_{G}^{2} \leq\left\|W_{0}\right\|_{G}^{2}+\sum_{i=0}^{n} h \alpha\left\|\sigma(E) w_{i}\right\|^{2} \\
+\sum_{i=0}^{n} h \delta \sum_{j=1}^{\left\lfloor\left(i+\sigma^{\prime}(1)\right) / m\right\rfloor} \beta_{2}^{j-1}\left\|\sigma(E) w_{i-j m}\right\|^{2}+h \\
+\frac{\left(\beta_{1}+\beta_{2}\right)^{2}}{\delta} \Gamma^{2} \sum_{i=0}^{n} \beta_{2}^{\left\lfloor\left(i+\sigma^{\prime}(1)\right) / m\right\rfloor} \\
+h^{2} \beta_{3} \gamma \sum_{i=0}^{n} \sum_{j=0}^{\left\lfloor\left(i+\sigma^{\prime}(1)\right) / m\right\rfloor} \beta_{2}^{j}\left(\sum_{k=1}^{m-1}\left\|\sigma(E) w_{i-j m-k}\right\|^{2}\right. \\
\left.+\frac{1}{2}\left\|\sigma(E) w_{i-j m}\right\|^{2}+\frac{1}{2}\left\|\sigma(E) w_{i-(j+1) m}\right\|^{2}\right) \\
+\left\|W_{0}\right\|_{G}^{2}+\sum_{i=0}^{n} h \alpha\left\|\sigma(E) w_{i}\right\|^{2}+\frac{h \delta}{1-\beta_{2}} \sum_{i=0}^{n} \| \sigma(E) \\
+\frac{w_{i-m} \|^{2}+\frac{h m}{\delta\left(1-\beta_{2}\right)}\left(\beta_{1}+\beta_{2}\right)^{2} \Gamma^{2}+\frac{h^{2} \beta_{3} \gamma m}{1-\beta_{2}}}{1-\beta_{2}} \\
\left.+\frac{\beta_{3} \gamma \tau}{1-\beta_{2}}\right)\left\|\sigma(E) w_{i}\right\|^{2}+\frac{\tau\left(\beta_{1}+\beta_{2}\right)^{2}}{\delta\left(1-\beta_{2}\right)} \Gamma^{2} \\
+\sum_{i=-m}^{n}\left\|\sigma(E) w_{i}\right\|^{2} \leq\left\|W_{0}\right\|_{G}^{2}+\sum_{i=0}^{n} h\left(\alpha+\frac{\delta}{1-\beta_{2}}\right. \\
+\max _{-m \leq i \leq-1}\left\|\sigma(E) w_{i}\right\|^{2}+\frac{\tau\left(\beta_{1}+\beta_{2}\right)^{2}}{\delta\left(1-\beta_{2}\right)} \Gamma^{2}
\end{aligned}
$$

Let $\lambda_{1}$ and $\lambda_{2}$ denote the maximum and minimum eigenvalues of the matrix $G$, respectively. Then, we have

$$
\begin{aligned}
\lambda_{2}\left\|w_{n+k}\right\|^{2} \leq & \lambda_{1} \sum_{j=0}^{k-1}\left\|w_{j}\right\|^{2} \\
& +\frac{\delta+\beta_{3} \gamma \tau}{1-\beta_{2}} \tau \max _{-m \leq i \leq-1}\left\|\sigma(E) w_{i}\right\|^{2} \\
& +\frac{\tau\left(\beta_{1}+\beta_{2}\right)^{2}}{\delta\left(1-\beta_{2}\right)} \Gamma^{2} .
\end{aligned}
$$

Hence

$$
\begin{aligned}
\left\|w_{n+k}\right\|^{2} \leq & \frac{k \lambda_{1}}{\lambda_{2}} \max _{0 \leq j \leq k-1}\left\|w_{j}\right\|^{2} \\
& +\frac{\left(\delta+\beta_{3} \gamma \tau\right) \tau}{\left(1-\beta_{2}\right) \lambda_{2}} \max _{-m \leq i \leq-1}\left\|\sigma(E) w_{i}\right\|^{2} \\
& +\frac{\tau\left(\beta_{1}+\beta_{2}\right)^{2}}{\delta\left(1-\beta_{2}\right) \lambda_{2}} \Gamma^{2} .
\end{aligned}
$$

Therefore, there is a real constant $C$ depending only on the method, $\beta_{1}, \beta_{2}, \beta_{3}, \gamma, \delta$, and $\tau$ such that inequality (22) holds and this completes the proof of Theorem 6 .

Remark 7. It is well known that many one-leg methods, such as implicit Euler method, the second-order BDF formula method, and one-leg $\theta$-methods $(1 / 2 \leq \theta \leq 1)$, are all $A$ stable. Therefore, in terms of Theorem 6 , the corresponding methods are globally stable for solving the nonlinear NDIDEs of the class $\mathrm{D}\left(\alpha, \beta_{1}, \beta_{2}, \beta_{3}, \gamma, \delta\right)$ which satisfies the condition $\alpha+\left(\delta+\beta_{3} \gamma \tau\right) /\left(1-\beta_{2}\right) \leq 0$.

Next, we further discuss the asymptotic stability of the one-leg method. One-leg method (15) is called strongly $A$ stable if it is $A$-stable and the modulus of any root of $\sigma(x)$ is strictly less than 1 .

Theorem 8. Assume that one-leg method (15) is strongly Astable. Then the numerical solutions $\left\{y_{n}\right\}$ and $\left\{z_{n}\right\}$, obtained by using corresponding method (16) to problems (1) and (13) which belong to the class $\mathrm{D}\left(\alpha, \beta_{1}, \beta_{2}, \beta_{3}, \gamma, \delta\right)$ with $\alpha+\left(\delta+\beta_{3} \gamma \tau\right) /(1-$ $\left.\beta_{2}\right)<0$, respectively, satisfy

$$
\lim _{n \rightarrow+\infty}\left\|y_{n}-z_{n}\right\|=0
$$

for allh $>0$. Relation (35) characterizes the asymptotic stability property of method (16).

Proof. In terms of the proof of Theorem 6, we have

$$
\begin{aligned}
\left\|W_{n+1}\right\|_{G}^{2} \leq & \left\|W_{0}\right\|_{G}^{2}+\sum_{i=0}^{n} h\left(\alpha+\frac{\delta+\beta_{3} \gamma \tau}{1-\beta_{2}}\right)\left\|\sigma(E) w_{i}\right\|^{2} \\
& +\frac{\tau\left(\beta_{1}+\beta_{2}\right)^{2}}{\delta\left(1-\beta_{2}\right)} \Gamma^{2} \\
& +\frac{\delta+\beta_{3} \gamma \tau}{1-\beta_{2}} \tau \max _{-m \leq i \leq-1}\left\|\sigma(E) w_{i}\right\|^{2}
\end{aligned}
$$


Since $\alpha+\left(\delta+\beta_{3} \gamma \tau\right) /\left(1-\beta_{2}\right)<0$, it is easily obtained from (36) that

$$
\lim _{n \rightarrow+\infty}\left\|\sigma(E)\left(y_{n}-z_{n}\right)\right\|=0 .
$$

By analogy with the proof of Theorem 4.3 in [27], we have

$$
\lim _{n \rightarrow+\infty}\left\|y_{n}-z_{n}\right\|=0 \text {, }
$$

and this completes the proof of Theorem 8 .

Remark 9. It is well known that many one-leg methods, such as implicit Euler method, the second-order BDF formula method, and one-leg $\theta$-methods $(1 / 2<\theta \leq 1)$, are all strongly $A$-stable. Therefore, in terms of Theorem 8 , the corresponding methods are asymptotically stable for solving the nonlinear NDIDEs of the class $\mathrm{D}\left(\alpha, \beta_{1}, \beta_{2}, \beta_{3}, \gamma, \delta\right)$ which satisfies the condition $\alpha+\left(\delta+\beta_{3} \gamma \tau\right) /\left(1-\beta_{2}\right)<0$.

\section{Numerical Experiments}

Example 1. Consider the one-dimensional parabolic problem with neutral type

$$
\begin{aligned}
\frac{\partial u(x, t)}{\partial t}= & \frac{\partial^{2} u(x, t)}{\partial x^{2}} \\
& +0.01 \sin \left(u(x, t-1)+\frac{\partial u(x, t-1)}{\partial t}\right) \\
& +\int_{t-1}^{t} u(x, s) d s, \quad 0<x<1, t \geq 0, \\
u(x, t)= & \left(x-x^{2}\right) e^{-t}, \quad 0<x<1,-1 \leq t \leq 0, \\
u(0, t)= & u(1, t)=0 .
\end{aligned}
$$

After application of the numerical method of lines, we obtain the following NDIDEs:

$$
\begin{aligned}
u_{i}^{\prime}(t)= & \frac{1}{\Delta x^{2}}\left(u_{i-1}(t)-2 u_{i}(t)+u_{i+1}(t)\right) \\
& +0.01 \sin \left(u_{i}(t-1)+u_{i}^{\prime}(t-1)\right) \\
& \quad+\int_{t-1}^{t} u_{i}(s) d s, \quad t \geq 0 \\
u_{0}(t)= & u_{N}(t) \equiv 0, \quad t \geq 0, \\
u_{i}(t)= & i \Delta x(1-i \Delta x) e^{-t}, \quad t \leq 0, \quad i=1,2, \ldots, N-1,
\end{aligned}
$$

where $\Delta x$ is the spatial step, $N$ is a natural number such that $N \Delta x=1, x_{i}=i \Delta x, i=1,2, \ldots, N-1$, and $u_{i}(t)=u\left(x_{i}, t\right)$. Then, problem (40) belongs to the class $\mathrm{D}\left(\alpha, \beta_{1}, \beta_{2}, \beta_{3}, \gamma, \delta\right)$ with

$$
\begin{aligned}
& \alpha=-4 N^{2} \sin ^{2} \frac{\pi}{2 N}, \\
& \beta_{1}=\beta_{2}=0.01, \\
& \beta_{3}=\gamma=1, \\
& \delta=0.01\left(1+4 N^{2}\right),
\end{aligned}
$$

where the inner product is standard inner product. We take $\Delta x=0.1$ for the numerical method of lines; thus the condition $\alpha+\left(\delta+\beta_{3} \gamma \tau\right) /\left(1-\beta_{2}\right) \leq 0(<0)$ is satisfied, which means the analytical solution of problem (40) is stable and asymptotically stable.

We use the 2-step one-leg methods of order 2:

$$
\begin{aligned}
& \frac{3}{2} y_{n+2}-2 y_{n+1}+\frac{1}{2} y_{n} \\
& \quad=h f\left(t_{n+2}, \frac{5}{4} y_{n+2}-\frac{1}{2} y_{n+1}+\frac{1}{4} y_{n}\right),
\end{aligned}
$$

which is $A$-stable and strongly $A$-stable, for solving problem (40) and its perturbed problem, where the initial function of the perturbed problem is $v_{i}(t)=i \Delta x(1-i \Delta x) e^{-t}+$ $5 \sin t,-1 \leq t \leq 0, i=1,2, \ldots, 9$.

As a comparison, we also use the 2-step one-leg method of order 3:

$$
\begin{aligned}
y_{n+2} & -y_{n+1} \\
= & h f\left(t_{n}+\frac{3}{2} h, \frac{5}{12} y_{n+2}+\frac{8}{12} y_{n+1}-\frac{1}{12} y_{n}\right),
\end{aligned}
$$

which is not $A$-stable, for solving problem (40) and its perturbed problem. We denote the numerical solutions of problem (40) and its perturbed problems $u_{n}$ and $v_{n}$, where $u_{n}$ and $v_{n}$ are approximations to $\left[u_{1}\left(t_{n}\right), u_{2}\left(t_{n}\right), \ldots, u_{9}\left(t_{n}\right)\right]^{T}$ and $\left[v_{1}\left(t_{n}\right), v_{2}\left(t_{n}\right), \ldots, v_{9}\left(t_{n}\right)\right]^{T}$, respectively. The values $\left\|u_{n}-v_{n}\right\|$ obtained by different methods are listed in Figure 1.

Example 2. Consider the following neutral delay integrodifferential equations, which are the models for population dynamics (see [1]):

$$
\begin{aligned}
U^{\prime}(t)= & b_{0} U(t)+b_{1} V(t)+b_{2} U(t-\tau)+b_{3} V(t-\tau) \\
& +b_{4} V^{\prime}(t-\tau) \\
& +\int_{t-\tau}^{t} k_{1}(t, s, U(s), V(s)) d s \\
V^{\prime}(t)= & c_{1} V(t)+c_{2} U(t-\tau)+c_{3} V(t-\tau) \\
& +c_{4} V^{\prime}(t-\tau) \\
& +\int_{t-\tau}^{t} k_{2}(t, s, U(s), V(s)) d s, \quad t>0 \\
U(t)= & \varphi(t) \\
V(t)= & \psi(t)
\end{aligned}
$$




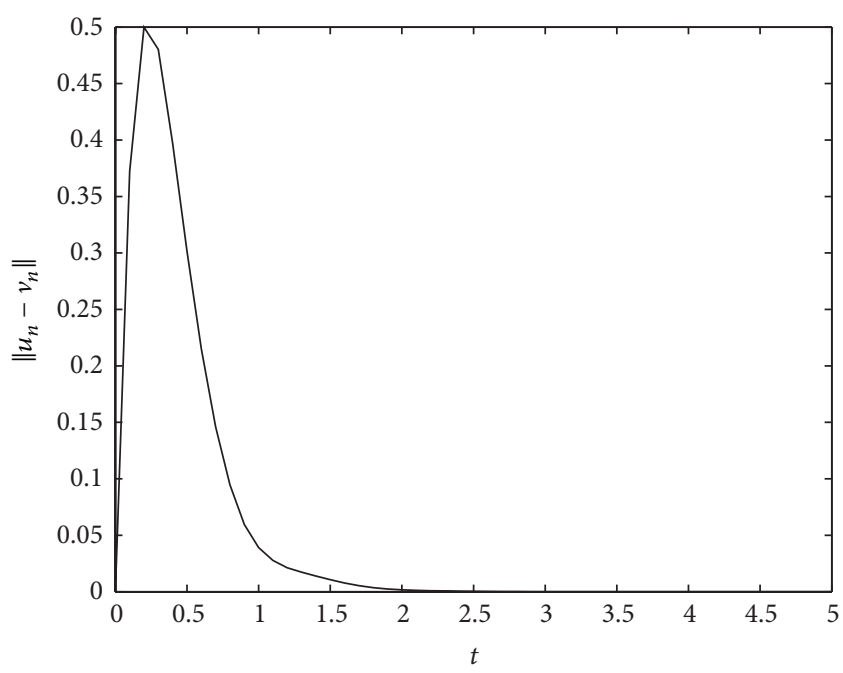

(a)

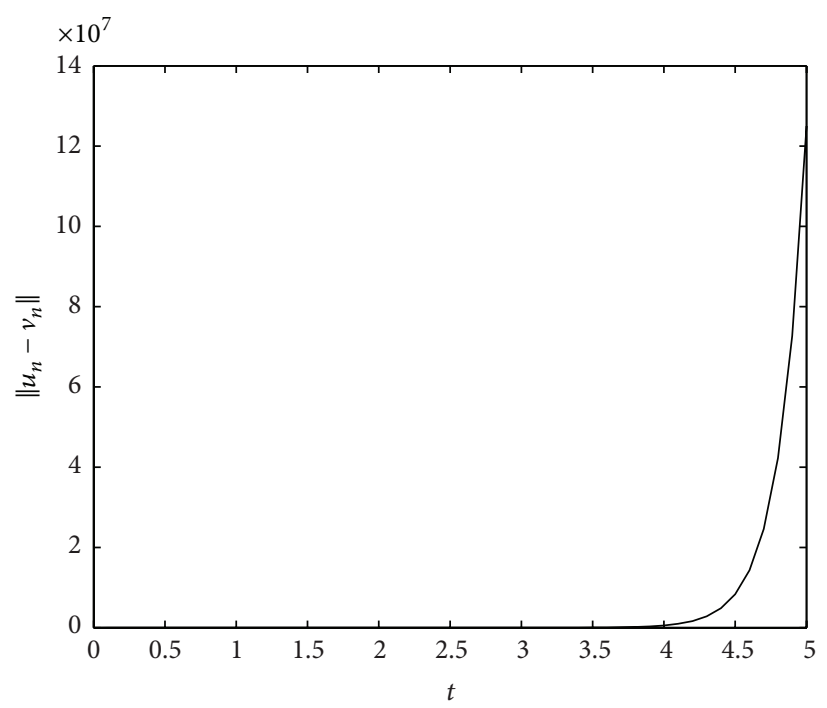

(b)

FIgURE 1: Values $\left\|u_{n}-v_{n}\right\|$ obtained by method (42) (a) or by method (43) (b) with $h=0.1$.

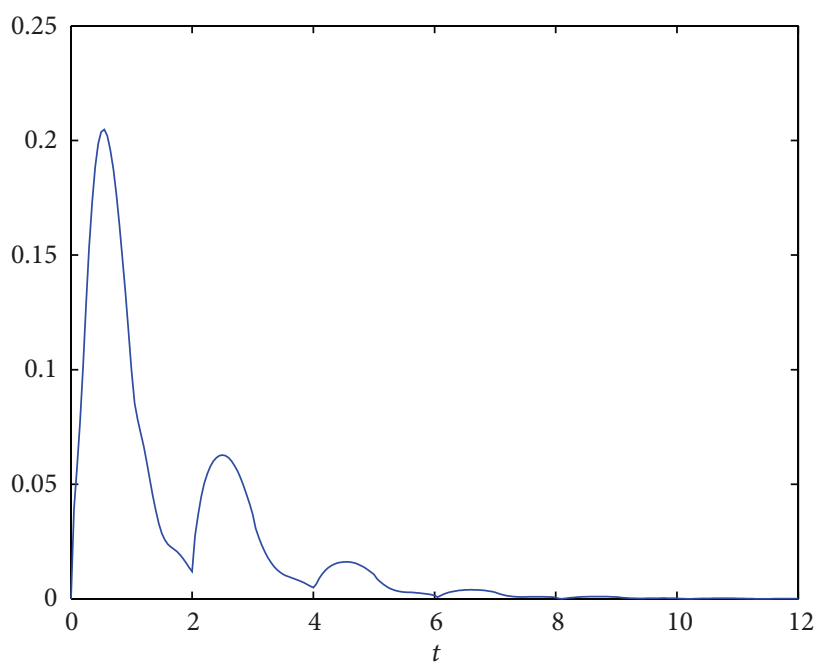

(a)

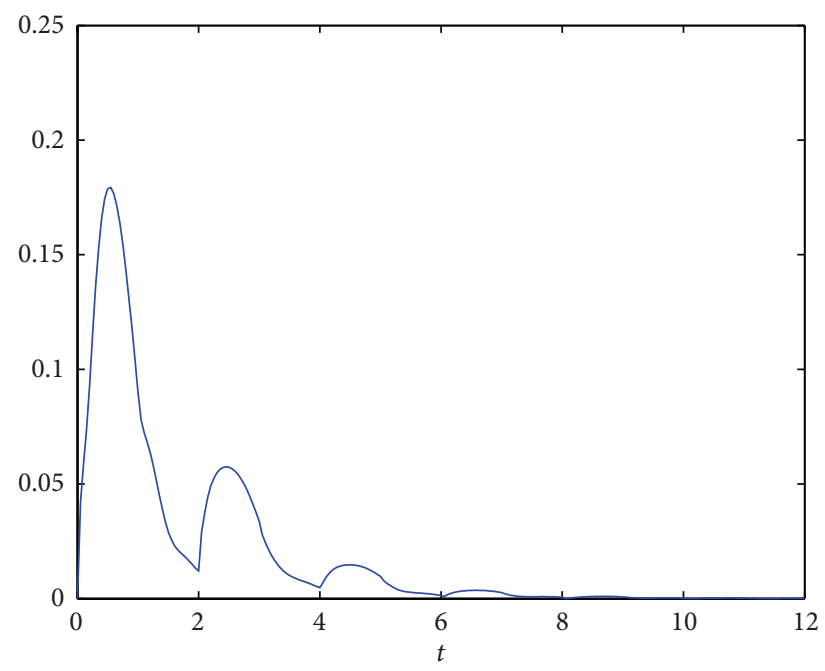

(b)

FIGURE 2: Values $\left\|W_{n}-\widetilde{W}_{n}\right\|$ obtained by method (42) with $h=0.05$ (a) and $h=0.02$ (b).

We choose $b_{0}=-4, b_{1}=1, b_{2}=1, b_{3}=-0.5, b_{4}=$ $0.1, c_{1}=-10, c_{2}=0.5, c_{3}=-1, c_{4}=0.05, k_{1}(t, s, U, V)=$ $0.5 U(s) \sin s, k_{2}(t, s, U, V)=0.5 \mathrm{~V}(s) \cos s$, and $\tau=1$. Then, problem (44) belongs to the class $\mathrm{D}\left(\alpha, \beta_{1}, \beta_{2}, \beta_{3}, \gamma, \delta\right)$ with

$$
\begin{aligned}
& \alpha \approx-3.84, \\
& \beta_{1}=1.5, \\
& \beta_{2} \approx 0.112, \\
& \beta_{3}=0.5 \\
& \gamma=1, \\
& \delta \approx 2.622 .
\end{aligned}
$$

Thus the condition $\alpha+\left(\delta+\beta_{3} \gamma \tau\right) /\left(1-\beta_{2}\right) \leq 0(<0)$ is satisfied, which means the analytical solution of problem (44) is stable and asymptotically stable. We use the 2-step one-leg method (42) for solving problem (44) and its perturbed problem, where the initial functions of problem (44) and its perturbed problem are $\varphi(t)=1.1, \psi(t)=0.9$ and $\widetilde{\varphi}(t)=1.1-\sin (\pi / 2) t$, $\widetilde{\psi}(t)=0.9-\sin (\pi / 2) t$ for $-1 \leq t \leq 0$, respectively. We denote the numerical solutions of problem (44) and its perturbed problems $W_{n}$ and $\widetilde{W}_{n}$, where $W_{n}$ and $\widetilde{W}_{n}$ are approximations to $\left[U\left(t_{n}\right), V\left(t_{n}\right)\right]^{T}$ and $\left[\widetilde{U}\left(t_{n}\right), \widetilde{V}\left(t_{n}\right)\right]^{T}$, respectively. The values $\left\|W_{n}-\widetilde{W}_{n}\right\|$ obtained by method (42) with different stepsize $h$ are listed in Figure 2.

From Figures 1-2, one can see that the values $\left\|u_{n}-v_{n}\right\|$ (or $\left.\left\|W_{n}-\widetilde{W}_{n}\right\|\right)$ obtained by method (42) are bounded and tend 
to zero. These coincide with Theorems 6 and 8 . However, for method (43), the situation is inverse as one can see that the values $\left\|u_{n}-v_{n}\right\|$ are divergent as $n \rightarrow+\infty$.

\section{Conclusion}

In the present paper, the adaptation of one-leg methods is applied for solving nonlinear NDIDEs. It is proved that an $A$-stable one-leg method can preserve the global stability and a strongly $A$-stable one-leg method can preserve the asymptotic stability of the analytical solution of nonlinear NDIDEs. Investigating the stability of other numerical methods, for example, multistep Runge-Kutta methods, for solving nonlinear NDIDEs will be our future work.

\section{Conflict of Interests}

The authors declare that there is no conflict of interests regarding the publication of this paper.

\section{Acknowledgments}

The authors would like to thank an anonymous referee for his many valuable suggestions. This work was supported by the NSF of China $(11171282,11371302)$ and Scientific Research Fund of Hunan Provincial Education Department.

\section{References}

[1] G. A. Bocharov and F. A. Rihan, "Numerical modelling in biosciences using delay differential equations," Journal of Computational and Applied Mathematics, vol. 125, no. 1-2, pp. 183$199,2000$.

[2] J. K. Hale and S. M. V. Lunel, Introduction to Functional Differential Equations, Springe, Berlin, Germany, 1993.

[3] V. Kolmanovskii and A. Myshkis, Introduction to the Theory and Applications of Functional Differential Equations, Kluwer Academic, Dordrecht, The Netherlands, 1999.

[4] J. J. Zhao, Y. Xu, and M. Z. Liu, "Stability analysis of numerical methods for linear neutral Volterra delay-integro-differential system," Applied Mathematics and Computation, vol. 167, no. 2, pp. 1062-1079, 2005.

[5] Y. Xu and J. J. Zhao, "Stability of Runge-Kutta methods for neutral delay-integro-differential-algebraic system," Mathematics and Computers in Simulation, vol. 79, no. 3, pp. 571-583, 2008.

[6] C. Zhang and S. Vandewalle, "Stability criteria for exact and discrete solutions of neutral multidelay-integro-differential equations," Advances in Computational Mathematics, vol. 28, no. 4, pp. 383-399, 2008.

[7] S. F. Wu and S. Q. Gan, "Analytical and numerical stability of neutral delay integro-differential equations and neutral delay partial differential equations," Computers \& Mathematics with Applications, vol. 55, no. 11, pp. 2426-2443, 2008.

[8] Y.-X. Yu and S.-F. Li, "Stability analysis of Runge-Kutta methods for nonlinear neutral delay integro-differential equations," Science in China-Series A: Mathematics, vol. 50, no. 4, pp. 464474, 2007.

[9] P. Hu and C. M. Huang, "Analytical and numerical stability of nonlinear neutral delay integro-differential equations," Journal of the Franklin Institute, vol. 348, no. 6, pp. 1082-1100, 2011.
[10] Y. X. Yu, L. P. Wen, and S. F. Li, "Nonlinear stability of RungeKutta methods for neutral delay integro-differential equations," Applied Mathematics and Computation, vol. 191, no. 2, pp. 543549, 2007.

[11] C. J. Zhang, T. T. Qin, and J. Jin, "An improvement of the numerical stability results for nonlinear neutral delay-integrodifferential equations," Applied Mathematics and Computation, vol. 215, no. 2, pp. 548-556, 2009.

[12] C. J. Zhang and Y. Y. He, "The extended one-leg methods for nonlinear neutral delay-integro-differential equations," Applied Numerical Mathematics, vol. 59, no. 6, pp. 1409-1418, 2009.

[13] T. Koto, "Stability of Runge-Kutta methods for delay integrodifferential equations," Journal of Computational and Applied Mathematics, vol. 145, no. 2, pp. 483-492, 2002.

[14] H. Brunner and R. Vermiglio, "Stability of solutions of delay functional integro-differential equations and their discretizations," Computing. Archives for Scientific Computing, vol. 71, no. 3, pp. 229-245, 2003.

[15] C. J. Zhang and S. Vandewalle, "Stability analysis of RungeKutta methods for nonlinear Volterra delay-integro-differential equations," IMA Journal of Numerical Analysis, vol. 24, no. 2, pp. 193-214, 2004.

[16] C. J. Zhang and S. Vandewalle, "General linear methods for Volterra integro-differential equations with memory," SIAM Journal on Scientific Computing, vol. 27, no. 6, pp. 2010-2031, 2006.

[17] C. M. Huang, "Stability of linear multistep methods for delay integro-differential equations," Computers \& Mathematics with Applications, vol. 55, no. 12, pp. 2830-2838, 2008.

[18] G.-D. Hu and T. Mitsui, "Stability analysis of numerical methods for systems of neutral delay-differential equations," BIT Numerical Mathematics, vol. 35, no. 4, pp. 504-515, 1995.

[19] Y. Liu, "Numerical solution of implicit neutral functionaldifferential equations," SIAM Journal on Numerical Analysis, vol. 36, no. 2, pp. 516-528, 1999.

[20] A. Bellen, N. Guglielmi, and M. Zennaro, "Numerical stability of nonlinear delay differential equations of neutral type," Journal of Computational and Applied Mathematics, vol. 125, no. 1-2, pp. 251-263, 2000.

[21] R. Vermiglio and L. Torelli, "A stable numerical approach for implicit non-linear neutral delay differential equations," BIT Numerical Mathematics, vol. 43, no. 1, pp. 195-215, 2003.

[22] W.-S. Wang, S.-F. Li, and K. Su, "Nonlinear stability of RungeKutta methods for neutral delay differential equations," Journal of Computational and Applied Mathematics, vol. 214, no. 1, pp. 175-185, 2008.

[23] L. Torelli, "Stability of numerical methods for delay differential equations," Journal of Computational and Applied Mathematics, vol. 25, no. 1, pp. 15-26, 1989.

[24] A. Bellen and M. Zennaro, "Strong contractivity properties of numerical methods for ordinary and delay differential equations," Applied Numerical Mathematics, vol. 9, no. 3-5, pp. 321346, 1992.

[25] K. J. In 't Hout, "Stability analysis of Runge-Kutta methods for systems of delay differential equations," IMA Journal of Numerical Analysis, vol. 17, no. 1, pp. 17-27, 1997.

[26] M. Zennaro, "Asymptotic stability analysis of Runge-Kutta methods for nonlinear systems of delay differential equations," Numerische Mathematik, vol. 77, no. 4, pp. 549-563, 1997.

[27] C. M. Huang, S. F. Li, H. Y. Fu, and G. N. Chen, "Stability and error analysis of one-leg methods for nonlinear delay 
differential equations," Journal of Computational and Applied Mathematics, vol. 103, no. 2, pp. 263-279, 1999.

[28] A. Bellen and M. Zennaro, Numerical Methods for Delay Differential Equations, Clarendon Press, Oxford, UK, 2003.

[29] G. Dahlquist, "G-stability is equivalent to A-stability," BIT Numerical Mathematics, vol. 18, no. 4, pp. 384-401, 1978. 


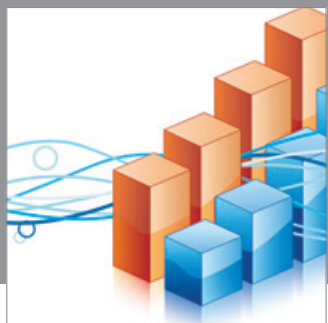

Advances in

Operations Research

mansans

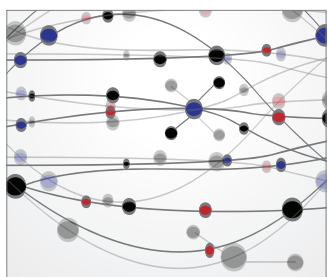

The Scientific World Journal
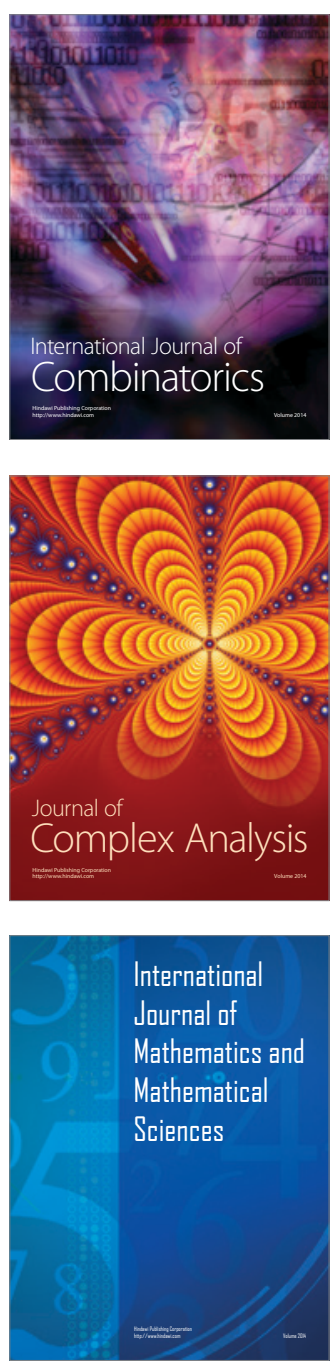
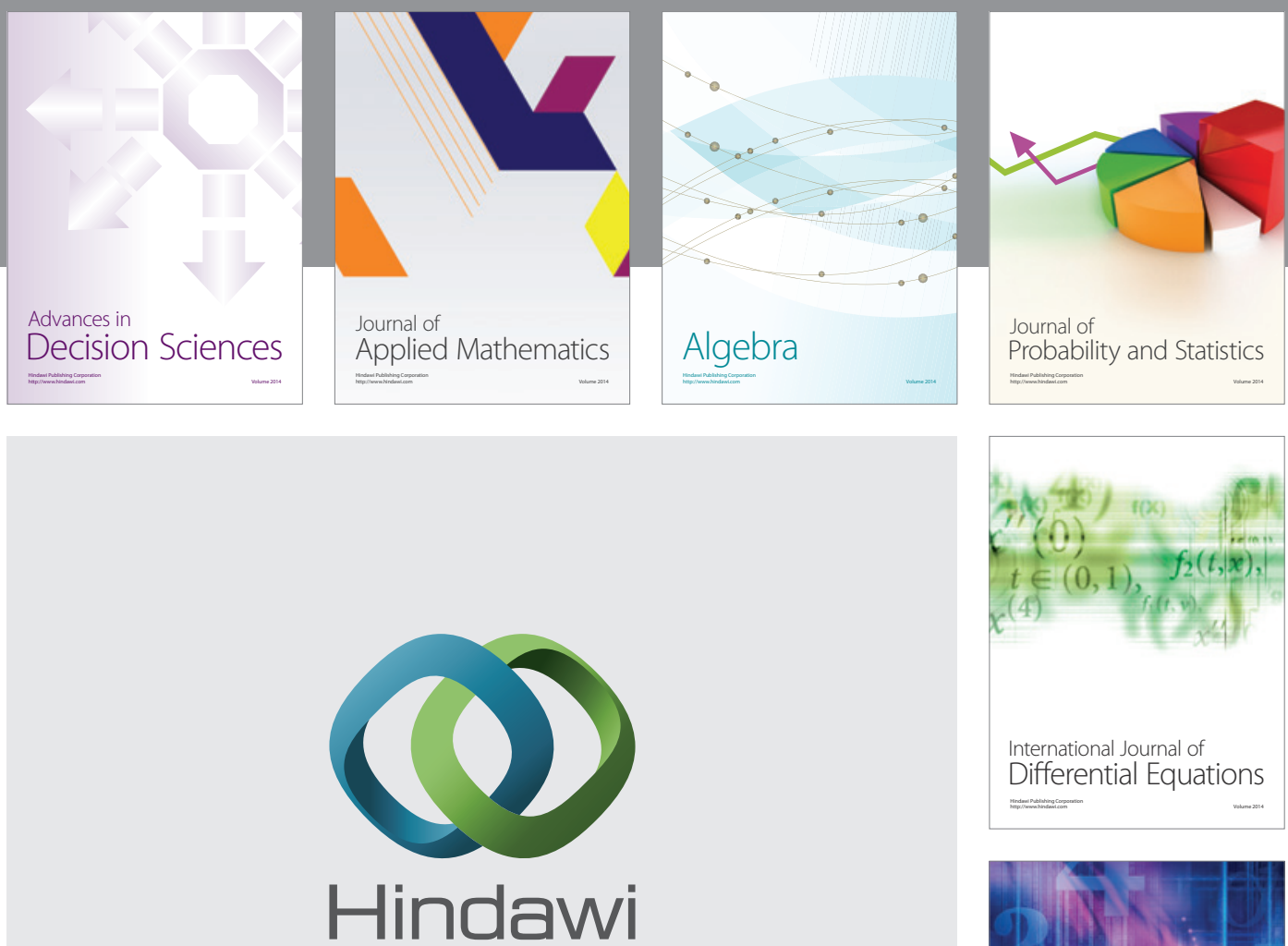

Submit your manuscripts at http://www.hindawi.com
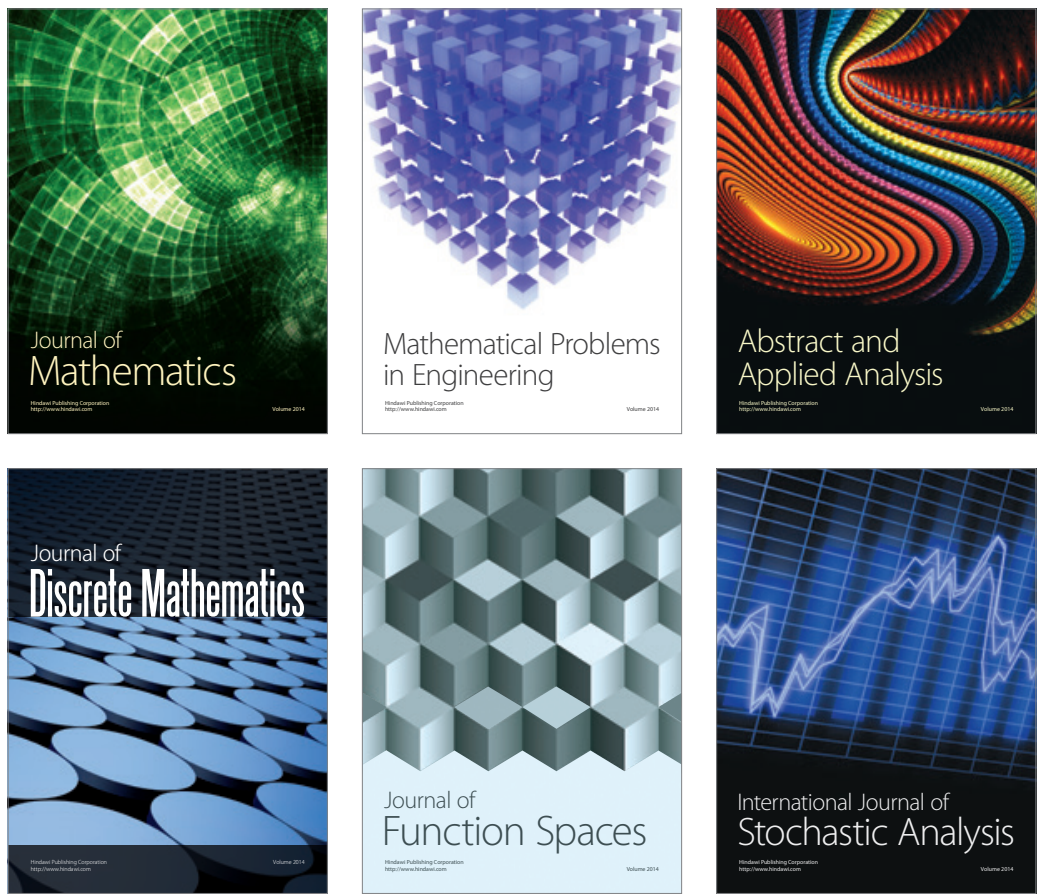

Journal of

Function Spaces

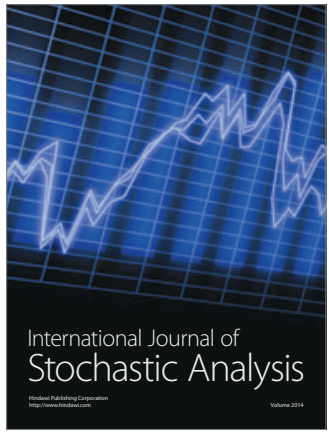

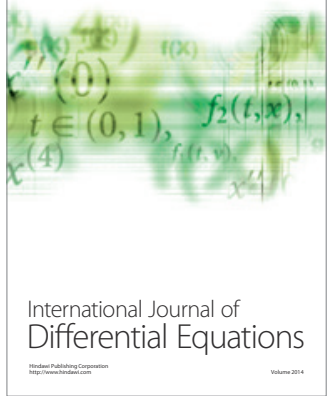
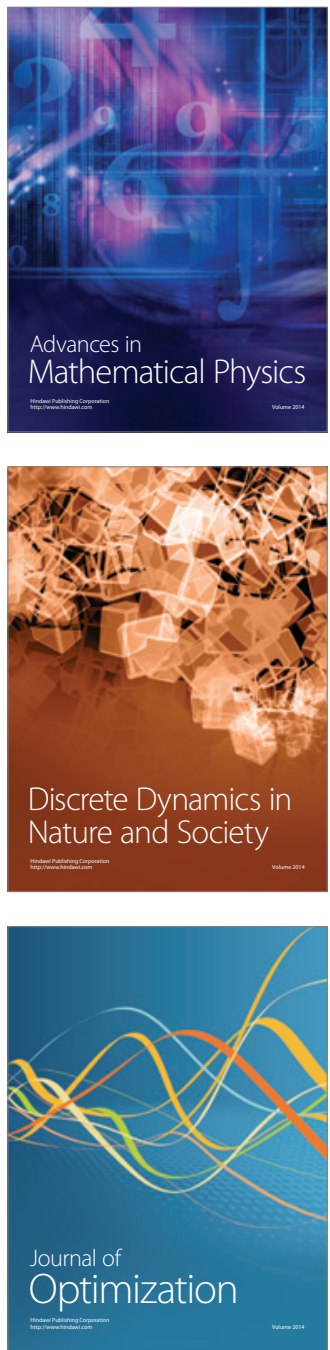